\title{
Validity and usefulness of the student-athletes' motivation toward sport and academics questionnaire: a Bayesian multilevel approach
}

\author{
Ricardo Quinaud ${ }^{1}$, Carlos Gonçalves $^{2}$, Kauana Possamai ${ }^{1}$, Cristiano Zarbato Morais $^{1}$, Laura Capranica ${ }^{3}$, \\ Humberto M. Carvalho ${ }^{\text {Corresp. } 1}$ \\ ${ }^{1}$ Department of Physical Education/ School of Sports, Universidade Federal de Santa Catarina, Florianópolis, Santa Catarina, Brazil \\ 2 Faculty of Sport Sciences and Physical Education, University of Coimbra, Coimbra, Portugal \\ 3 Department of Movement, Human and Health Sciences, University of Rome "Foro Italico", Rome, Italy \\ Corresponding Author: Humberto M. Carvalho \\ Email address: humberto.m.carvalho@ufsc.br
}

Background Reliable assessment and understanding of student-athletes' motivation for dual careers are crucial to support their career development and transitions. The purpose of this research was to examine the validity and usefulness of the student-athletes' motivation toward sport and academics questionnaire (SAMSAQ-PT) in the Brazilian higher education context. Four studies were performed.

Methods In study one, conceptually and semantic translation of the questionnaire and Bayesian exploratory factor analysis were conducted. In study two, a Bayesian confirmatory factor analysis with an independent sample was performed. In study three, Bayesian multilevel modeling was applied to examine the construct validity of the questionnaire in a cross-sectional sample. In study four, the SAMSAQ-PT sensitiveness was examined in a longitudinal sample, and the results were interpreted based on multilevel regression and poststratification.

Results Altogether the results provided evidence validity and usefulness of the SAMSAQ-PT in Brazilian student-athletes. The Brazilian student-athletes' motivation scores were sensitive to the influence of sex, sport level, and type of university on career and sport motivation. SAMSAQ-PT estimate scores across an academic year showed a trend of stability in the scores, adjusting for sex, sport level, type of university, and student-athlete status.

Conclusion The SAMSAQ-PT proved to be a robust and valuable questionnaire, which could be used in Portuguese-speaking countries. The findings of the cross-sectional and longitudinal surveys urge to consider individual and contextual characteristics when investigating motivation of dual-career of athletes, also concerning the sex-related opportunities in university sports. Furthermore, there is a need for a call for action to promote and nurture the student-athletes motivation to remain engaged in both sports and educational commitments. 


\section{Validity and usefulness of the Student-athletes'}

2 motivation toward sport and academics

3 questionnaire: a Bayesian multilevel approach

4

5

6

7

Ricardo T. Quinaud ${ }^{1}$, Carlos E. Gonçalves ${ }^{2}$, Kauana Possamai ${ }^{1}$, Cristiano Z. Morais ${ }^{1}$, Laura Capranica $^{3}$, Humberto M. Carvalho ${ }^{1}$

${ }^{1}$ Department of Physical Education/School of Sports, Universidade Federal de Santa Catarina, Florianópolis, Santa Catarina, Brazil

${ }^{2}$ Faculty of Sport Sciences and Physical Education, University of Coimbra, Coimbra, Portugal ${ }^{3}$ Department of Movement, Human and Health Sciences, University of Rome "Foro Italico", Rome, Italy

Corresponding Author:

Humberto M Carvalho ${ }^{1}$

Campus Reitor João David Ferreira Lima

88040-900 Florianópolis, Santa Catarina, Brasil

Email address: hmoreiracarvalho@gmail.com

\section{Abstract}

\section{Background}

Reliable assessment and understanding of student-athletes' motivation for dual careers are crucial to support their career development and transitions. The purpose of this research was to examine the validity and usefulness of the student-athletes' motivation toward sport and academics questionnaire (SAMSAQ-PT) in the Brazilian higher education context. Four studies were performed.

\section{Methods}

In study one, conceptually and semantic translation of the questionnaire and Bayesian exploratory factor analysis were conducted. In study two, a Bayesian confirmatory factor analysis with an independent sample was performed. In study three, Bayesian multilevel modeling was applied to examine the construct validity of the questionnaire in a cross-sectional sample. In study four, the SAMSAQ-PT sensitiveness was examined in a longitudinal sample, and the results were interpreted based on multilevel regression and poststratification.

\section{Results}

Altogether the results provided evidence validity and usefulness of the SAMSAQ-PT in Brazilian student-athletes. The Brazilian student-athletes' motivation scores were sensitive to the influence of sex, sport level, and type of university on career and sport motivation. SAMSAQ-PT estimate scores across an academic year showed a trend of stability in the scores, adjusting for sex, sport level, type of university, and student-athlete status. 


\section{Conclusion}

41 The SAMSAQ-PT proved to be a robust and useful questionnaire, which could be used in

42 Portuguese speaking countries. The findings of the cross-sectional and longitudinal surveys urge

43 to consider individual and contextual characteristics when investigating motivation of dual-

44 career of athletes, also in relation to the sex-related opportunities in university sports.

45 Furthermore, there is a need for call for actions to promote and nurture the student-athletes'

46 motivation to remain engaged in both sports and educational commitments.

47

48

49

50

51

52

53

54

55

56

57

58

59

60

61

62

63

64

65

66

67

68

69

70

71

72

73

74

75

76

77

78

79

\section{Introduction}

At the university level, student-athletes present several social, cultural, and individual challenges to pursue their sport and education paths (i.e., dual-career), especially at the start of the college degree (Aquilina, 2013; Condello et al., 2019; Gaston-Gayles \& Baker, 2015; Ryba et al., 2015; Simons et al., 1999). The attention in dual-career, defined as "a career with major focus on sport and study or work" (Stambulova \& Wylleman, 2015), has increased in the past years (Stambulova \& Wylleman, 2019). Considering the different sports and educational contexts and the various dual-career approaches in place in the European Member States, the European dualcareer recommendations urge strategies to foster the student-athletes motivation to pursue their academic and sports achievements (European Commission, 2012). Indeed, motivation is determinant to keep people involved in what they do (Ferdinand \& Czernochowski, 2018; Ryan et al., 2019). Thus, understanding student-athletes motivation for dual-career is crucial to support their career development and transitions (Stambulova et al., 2020). In general, dual-career pathways depend on student-athletes' motivation, identity, health, lifestyle, and wellbeing (Aunola et al., 2018; Breslin et al., 2019; Cartigny et al., 2019; Harrison et al., 2020; Lupo et al., 2017; Martin, 2005; Ryba et al., 2016, 2107; Sorkkila et al., 2018).

In studying the dual-career motivation of student-athletes from different cultures, the robustness of the psychometric instrument is crucial for cross-cultural comparisons and applied sport psychology (Joshanloo et al., 2014; Sullivan et al., 2020; Wu et al., 2014). Since the development of the Student-athletes' Motivation toward Sports and Academics Questionnaire (SAMSAQ) in the United States (Gaston-Gayles, 2005) and its validation tested in European (Guidotti \& Capranica, 2013; Lupo et al., 2012, 2015) and Asian (Park et al., 2015) contexts, the effects of different cultures and dual-career support policies have been hypothesized (Fernandes et al., 2019; Fortes et al., 2010; Gaston-Gayles \& Baker, 2015; Guidotti \& Capranica, 2013; Guidotti et al., 2015; Lupo et al., 2012, 2015; Park et al., 2015). However, a lack of knowledge for Latin America's countries is still present.

Brazil is the largest country in Latin America. In having a federal structure, Brazil presents contrasting demographic characteristics and cultural backgrounds (Hofstede et al., 2010). While the Brazilian regulation of sports at the federal level, including university sports, was established in 1941 (Brasil, 1941), rules and criteria for the allocation of public resources to the sports sector were established in 1998, assigning responsibility to sports organizations concerning the educational system and vice versa (Brasil, 1998). In particular, the Brazilian 
80 University Sports Confederation is responsible for organizing and developing university sports, 81 whereas the Ministry of Education has the primary responsibility of the sports policies allowing 82 athletes to combine their dual-career. Brazilian public higher education institutions enroll around 83 two million students per year (Instituto Nacional de Estudos e Pesquisas Educacionais Anísio 84 Teixeira, 2018). As for private higher education institutions, around six million students enroll 85 each year (Instituto Nacional de Estudos e Pesquisas Educacionais Anísio Teixeira, 2018). Public 86 universities often provide sports infrastructure for students and private universities offer financial 87 support for athletes. However, federal regulation of dual-career policy for student-athletes in 88 Brazil is still not warranted (Carvalho \& Hass, 2015). In the absence of a clear dual-career policy

89

90

91

92

93

94

95

96

97

98

99

100

101

102

103

104

105

106

107

108

109

110

111

112

113

114

115

116

117

118

119 in the Brazilian sports system, some sports areas tend to be privileged, and others may be left unattended, leaving gaps in public service coverage. Especially at the state and local levels, programs and actions appear to vary according to different political approaches (Houlihan, 2005). Therefore, differences among Brazilian states and the country's federalism structure could provide a different level of dual-career support and influence student-athletes' motivation (Guidotti et al., 2015).

There have been problems to replicate psychological results, also referred to as the crisis of confidence (Open Science Collaboration, 2015). With few exceptions (Schweizer \& Furley 2016), sports psychology has overlooked this debate. One of the general debate consequences has been the increased awareness of the limitations and inappropriateness of testing nullhypotheses, establishing statistical significance and p-value use (Amrhein \& Greenland 2018, McShane, et al. 2019). Indeed, psychology research deals with complex interactions, noisy measurements, often expected between-individuals heterogeneity, and non-representative and imbalanced samples. To account for different sources of inferential uncertainty, Bayesian methods allow combining the information known before seeing the data (i.e., the prior uncertainty concerning a parameter or hypothesis expressed as a probability distribution) with what is learned from the observed data (i.e., the likelihood of the data conditioned on the parameter or hypothesis) to update knowledge expressed as the posterior distribution (Kennedy \& Gelman, 2020; Lee \& Wagenmakers, 2013).

Furthermore, the analysis and interpretation in sports psychology research often deal with traditional single-level approaches, albeit with the limitations noted in several scientific areas (Gelman \& Shalizi 2013). A multilevel modeling framework provides a flexible alternative that intuitively considers the data structure and the different sources of variation, providing trustable estimations and predictions for a target population (Gelman \& Hill, 2007). The framework has been noted as valuable to advancing cross-culture studies in psychology (Van Hoorn, 2015). Another main advantage of multilevel modeling lies in the natural fit of repeated measures (Singer \& Willett, 2003). Considering the lack of longitudinal studies on student-athlete motivation (Stambulova \& Wylleman, 2019), multilevel modeling is highly recommended to improve our understanding of Brazilian student-athletes' motivation toward dual-career.

The present research made use of a Bayesian approach and conducted four studies to examine the validity of the Portuguese version of the harmonized Italian Student-athletes' 
120 Motivation toward Sports and Academics Questionnaire (SAMSAQ-IT/A; Guidotti \& Capranica,

121

122

123

124

125

126

127

128

129

130

131

132

133

134

135

136

137

138

139

140

141

142

143

144

145

146

147

148

149

150

151

152

153

154

155

156

157

158

159

2013) and to assess its usefulness in discriminating the influence of academic and sport contexts as sources of variation in the scores in cross-sectional and longitudinal research approaches. In study one, we aimed to translate and explore the psychometric structure of the Portuguese version of the SAMSAQ-IT/A (SAMSAQ-PT) using Bayesian exploratory factor analysis. In study two, we tested the factor structure of the questionnaire that emerged from study one by applying a Bayesian confirmatory factor analysis with an independent sample. Based on the evidence of construct validity, in study three, we aimed to examine the construct validity of the questionnaire in a cross-sectional sample considering sex, sport level, the student-athlete status, and the type of university attended applying Bayesian multilevel regression. Lastly, in study four, we aimed to explore the student-athletes' motivation scores' sensitiveness. Hence, we considered a longitudinal measure design across an academic year to analyze changes in motivation scores adjusted for sex, sport level, student-athlete status, and type of university.

\section{Research design}

The research ethics committee of the Federal University of Santa Catarina approved the present research ( $\left.n^{\circ} 2.949 .805\right)$ and voluntary student-athlete provided written consent to participate in the study. The inclusion criteria for recruiting participants encompassed: 1) to be enrolled in a higher education degree; and 2) to compete in organized sports of the Brazilian University Sports Confederation. Data were collected during the Santa Catarina University Games in July 2018 and 2019 (i.e., state Games) and the Brazilian University Games in November 2018 and October 2019 (i.e., national Games). The state games had about 800 athletes, while the national games had about 2.000 athletes participating. Studies one, two, and three comprised participants only from cross-sectional observations. Study four comprised participants from repeated observations across an academic year (measured in 2018 and 2019). Supplementary materials including data and code are available at https://osf.io/cpwdv/.

\section{Study one}

This study aimed to translate and explore the psychometric structure of the SAMSAQ-PT using Bayesian exploratory factor analysis. Independent forward and backward Portuguese translations of the SAMSAQ-IT/A (Guidotti \& Capranica, 2013) and cognitive interviews were performed to ensure a conceptually and semantic trustable instrument applied in the Brazilian context (Herdman et al., 1997; Su \& Parham, 2002). Then, the SAMSAQ-PT was administered to a subsample of 74 Brazilian university student-athletes who individually completed the 30 -item SAMSAQ-PT, indicating their level of agreement with the statements on a 6-point Likert scale, ranging from 1 (strongly disagree) to 6 (strongly agree). Interviews aiming to ascertain the reasons behind responses were performed to verify the instructions, items, and response options. Therefore, the SAMSAQ-PT was considered suitable to be administered to Brazilian studentathletes. 
160 Methods

161 Participants

162 The total sample comprises 862 student-athletes from all five regions of Brazil; however, about

$16372.6 \%$ were from the same region (south). The total sample was split into two independent

164 samples generated through random numbers. In study one, we used the first $50 \%$ of the dataset.

165 The sample comprised 248 female and 183 male student-athletes $\left(\mathrm{M}_{\text {age }}=21.7, \mathrm{SD}=3.4 \mathrm{yrs}\right)$

166 enrolled in public $(n=200)$ and private $(n=231)$ Brazilian universities and competing at

167 international $(n=33)$, national, $(n=131)$, state $(n=74)$ and university $(n=193)$ levels. Descriptive

168 analysis is presented in Supplementary Table 1 (available at https://osf.io/cpwdv/).

169

170

\section{Data analysis}

171

A Bayesian exploratory factor analysis (BEFA) was conducted, with an initial four latent factors

172 maximum (Kmax) constraint (Guidotti \& Capranica, 2013). Then, different factor-structures

173 testing different models were allowed if the original model factor-structure was not confirmed. A total of 60,000 iterations with a burn-in period of 5,000 iterations were run. Default identification

175 restriction ( $\mathrm{Nid}=1$ ) was used, which lies on the minimum number of manifest variables dedicated to each factor Metropolis-Hastings' acceptance rate was used to retain items' posterior probabilities of being different from zero. Considering it is an exploratory analysis, a minimum posterior mean of 3 (cut-off point) was set as an acceptable value to retain an item (Peeters, 2012). Although there is no established recommendation of the minimum acceptable value to retain an item in BEFA, we set it as 3 for convenience (representing a closer interpretation of the frequentist factor loading "0.3"). Thus, items with factor values lower than three were excluded. The BEFA estimates were obtained using the "BayesFM" package (Conti et al., 2014), available as a package in the $\mathrm{R}$ statistical language (R Core Team, 2018).

\section{Results}

The SAMSAQ-PT presented a three-factor structure (Table 1). Due to low factor loadings $(<3)$, items 11 (2.74), 18 (1.92), 25 (2.60), and 30 (1.92) were excluded. In particular, seven items loaded the factor named "Sport Motivation" (SM), sixteen items loaded the factor named "Academic Motivation" (AM), and three items loaded the factor named "Career Motivation" 190 (CM).

191

192

193

194

195

196

197

198

199

[Insert Table 1 about here]

\section{Discussion}

Considering the translation and psychometric structure of the SAMSAQ-PT, we observed a three-factor structure. This structure is in line with the original American and the versions validated for Emirati, European, and Korean student-athletes (Gaston-Gayle, 2005; Fortes et al., 2010; Lupo et. al., 2015; Park et al., 2015). In particular, SM items express the desire for sports performance and for continuing sports career (Keshtidar \& Behzadnia, 2017), which is 
200 influenced by cultural and educational context for pursuing a sports performance (Fernandes et 201 al., 2019; Lupo et al. 2015); the AM items mirror the academic commitment of student-athletes, 202 measuring the Brazilian student-athletes' engagement in an educational/ vocational path 203 concerning a sport or a dual-career path (Cartigny et. al., 2019), probably considering the 204 academic degree relevant for future jobs and professional careers (Fortes et al., 2010); and the $205 \mathrm{CM}$ items present a desire for developing a professional sports career (Gaston-Gayles, 2005). 206 Thus, it could be speculated that the Brazilian student-athletes may perceive sport and academic 207 commitments separately.

208

\section{Study two}

210 The purpose of study two was to test the 26-item of the 3-factor structure questionnaire emerging 211 from study one by applying a Bayesian confirmatory factor analysis with an independent sample.

212 Specifically, it was intended to provide evidence to allow an informed generalization of the 213 questionnaire factor structure.

214

\section{Methods}

\section{Participants}

217 The participants considered in this study were the other half of the total cross-sectional sample.

218 In particular, 242 female and 99 male student-athletes $\left(\mathrm{M}_{\mathrm{age}}=21.6, \mathrm{SD}=3.5 \mathrm{yrs}\right)$ enrolled in 219 public $(\mathrm{n}=201)$ and private $(\mathrm{n}=230)$ Brazilian universities and competing at international $(\mathrm{n}=26)$, 220 national, $(\mathrm{n}=139)$, state $(\mathrm{n}=86)$ and university $(\mathrm{n}=180)$ levels.

221

222

223

224

225

226

227

228

229

230

231

232

233

234

235

236

237

238

239

\section{Data analysis}

A Bayesian Confirmatory Factor Analysis (BCFA) was applied to examine the model factorial structure. Two chains for 10,000 iterations with 2,000 used as warm-up, using probabilistic programming language Stan (Carpenter et al., 2017). The model estimations were regularized using normal prior $(0,10)$ for the manifest variable (intercept) and normal prior $(0,1)$ for the latent variable were set. A posterior latent variable closer to 0.5 (Merkle \& Rossel, 2018) was set as a satisfactory value to retain an item based on the literature. Moreover, Bayesian root mean square error of approximation (BRMSEA), Bayesian Gamma Hat (BGammaHat), Adjusted Bayesian Gamma Hat (adjBgammahat), and Bayesian McDonald's centrality index (BMc) were also applied to confirm the model fit (Montenegro-Montenegro, 2020). BRMSEA value close to 0.5 and BGammaHat, adj BGammaHat and BMc values close to one indicate a better fit. The BCFA was conducted using the "blavaan" package (Merkle \& Rossel, 2018) in the R software (R Core Team, 2018).

\section{Results}

In the BCFA, items $1(0.28), 10(0.45), 12(0.47), 14(0.25)$ and $21(0.38)$ presented posterior values lower than 0.5 and were excluded. Additionally, this first model did not present good evidence of model fit $(\mathrm{BRMSEA}=0.08 ; \mathrm{BGammaHat}=0.87$; $\operatorname{adj} \mathrm{Bgammahat}=0.84 ; \mathrm{BMc}=$ 
240 0.43). The new model (21 items) was further tested and four items [items 5 (0.30), 17 (0.35), 24

241 (0.33), and 29 (0.39)] with low factor load values were excluded. In this model, fit indexes were 242 superior, but not well adequate $(B R M S E A=0.07$; $\mathrm{BGammaHat}=0.93$; adjBgammahat $=0.89$;

$243 \mathrm{BMc}=0.67)$. Thus, the resulting 17 -item model presented factor loadings close or above 0.5 for 244 all items (Supplementary table 2, available at https://osf.io/cpwdv/). Only item 13 presented 245 factor loading (0.49) bellow 0.5. Thus, the item was retained. Additionally, fit indexes showed 246 satisfactory adequacy $(\mathrm{BRMSEA}=0.06 ; \mathrm{BGammaHat}=0.96$; adjBgammahat $=0.93 ; \mathrm{BMc}=$ $2470.84)$.

\section{Discussion}

251

252

253

254

255

256

257

258

259

260

261

262

263

264

265

266

267

268

269

270

271

272

273

274

275

276

277

278

In this study, we tested the factor structure of SAMSAQ-PT, which emerged from study one by applying BCFA. Our observations confirmed the three-factor structure but indicated a better structure composed of 17 items for SM ( 7 items), AM ( 7 items), and CM ( 3 items). The confirmatory factor analysis of the SAMSAQ-PT substantiated the three-factor structure (Fortes et al., 2010; Gaston-Gayles, 2005; Guidotti et al., 2013; Lupo et al., 2015; Park et al., 2015), even though to avoid misinterpretations, the Bayesian inference based on strong similarities determined the substantial reduction of the item's distribution. Different factor structures can be expected due to dual-career policies and social-cultural contexts, as well as to the sport and educational levels of student-athletes (Fortes et al., 2010; Gaston-Gayles, 2005; Guidotti et al., 2013; Lupo et al., 2015; Lupo et al., 2017; Park, Hong, \& Lee, 2015; Stambulova \& Alfermann, 2009). Given the Brazilian miscegenation and the potential influence of European and other cultures on Brazilian student-athletes, both similarities and differences with other cultures were expected.

Regarding the similarities, the AM dimension presented stable comparing to other SAMSAQ validations (Gaston-Gayles, 2005; Guidotti et al., 2013; Lupo et al., 2015; Lupo et al., 2017; Park, Hong, \& Lee, 2015), although in the present version we had a higher reduction of items. The results may indicate that the academic motivation of higher education student-athletes is a drive based on similar purposes, such as learning (item 3), get good grades (item 4), and the course content (item 28). There were divergences, such as item 16 (the achievement of a degree is important to enrich my knowledge) had loaded in a different dimension than the academic motivation (Lupo et al., 2017). However, this item has a clear academic motivation.

Our results were consistent with the other validations in the SM dimension, but there is less agreement about the items from this dimension across the validation studies (Lupo et al., 2015; Lupo et al., 2017; Gaston-Gayles, 2005; Park, Hong, \& Lee, 2015). The feeling of being better than other athletes (item 13) is an example of how it can vary between the validations. It represents a sport and career motivation (Lupo et al., 2017), sport motivation (federation structure; Lupo et al., 2015; Gaston-Gayles, 2005), or even academic motivation (Park, Hong, \& Lee, 2015). In summary, there was a shift of items in SM and CM dimensions in the factor structure of the SAMSAQ-PT. The items with explicit statements focused on professional or

Peer) reviewing PDF | (2021:03:58830:2:0:NEW 30 Jun 2021) 
279

280

281

282

283

284

285

286

287

288

289

290

291

292

293

294

295

296

297

298

299

300

301

302

303

304

305

306

307

308

309

310

311

312

313

314

athletic careers loaded in the CM. The results suggest that Brazilian student-athletes interpret their motivation for a high level of performance or athletic career as a priority.

\section{Study three}

In this study, we aimed to examine the construct validity of the questionnaire in a cross-sectional sample considering sex, sport level, student-athlete status, and the type of university. In particular, the student-athlete status was considered as any document from the higher education institution that guarantees support for student-athletes (e.g., flexible exam schedule when representing their university or their country in competitions).

\section{Participants}

The whole sample of 862 student-athletes (females: 56.8\%; males: 43.4\%) enrolled in public $(\mathrm{n}=401)$ and private $(\mathrm{n}=461)$ Brazilian universities and competing at international $(\mathrm{n}=59)$, national $(n=270)$, state $(n=160)$ and university $(n=373)$ levels participated in this study.

\section{Data analysis}

Multilevel regression models were used to estimate SAMSAQ-PT and its dimensions among Brazilian student-athletes when grouped by sex (e.g., female and male), sports level (e.g., international, national, state and university), type of university (e.g., public and private) and student-athletes' status (e.g., yes and no). The multilevel models estimate the individual scores relying on the available information of individuals characteristics and using additional "random" predictors such as group or context characteristics (for individual i, with indexes, $s, a$, $l$, and $u$ for, sex, student-athlete status, sport level, and type of university, respectively). In Bayesian terms, these "random" or "group-level" effects are related to each other by their grouping structure, and the individuals' responses are partially pooled towards the group mean (Gelman \& Hill, 2007), as follows:

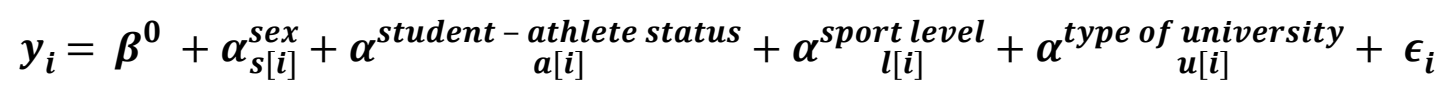

$$
\begin{aligned}
& \alpha_{s[i]}^{s e x} \sim N\left(0, \sigma_{s e x}^{2}\right), \text { for } s=1,2 .
\end{aligned}
$$

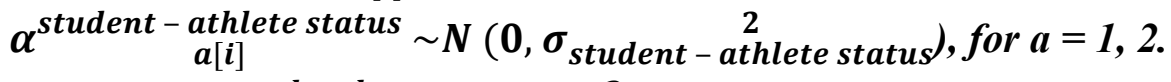

$$
\begin{aligned}
& \alpha_{l[i]}^{\text {sport level }} \sim N\left(0, \sigma_{\text {sport level }}^{2}\right) \text {, for } l=1,2,3,4 . \\
& \alpha^{\text {type of university }} \sim N\left(0, \sigma_{\text {type of university }}\right), \text { for } u=1,2 . \\
& \epsilon_{i} \sim N\left(0, \sigma_{y_{i}}^{2}\right)
\end{aligned}
$$

Weakly informative prior distributions, normal prior $(0,10)$ for population-level effect (intercept), and normal priors $(0,1)$ for group-level effects (i.e., the standard deviations of varying intercepts) to regularize the multilevel model estimations were used. Two chains for 4,000 iterations with a warm-up length of 1,000 iterations to ensure convergence of the Markov 
315 chains were run. To check the models and estimations, trace plots to examine the convergence of 316 Markov chains and posterior predictive checks to validate the models were used (Gelman et al., 317 2013). The Bayesian multilevel models were fitted with the "brms" package (Bürkner, 2017), 318 available as a package in the $\mathrm{R}$ statistical language (R Core Team, 2018). The brms package 319 implements Bayesian multilevel models using the probabilistic language Stan (Carpenter et al., 320 2017). For computational and interpretation convenience, the outcomes were standardized (z321 scores). This methodology is also described in previous studies (Quinaud, Fernandes, Gonçalves, 322 Carvalho, 2020; Quinaud, Gonçalves, Capranica, Carvalho, 2020).

323

324

\section{Results}

325

Figures 1, 2, and 3 present the standardized values of the SAMSAQ-PT's dimensions related to

326 the respondents' sex, type of universities, sport levels, and student-athletes' status. Male student-

327

328

329

330

331

332

333

334

335

336

337

338

339

340

341

342

343

344

345

346

347

348

349

350

351

352

353

354

athletes presented higher effect scores for SM and CM dimensions. On the other hand, female student-athletes presented higher scores for AM, albeit with a small magnitude. Student-athletes from private universities had substantially higher scores than student-athletes from public universities for SM, AM, and CM dimensions, but at best, the magnitude was small for AM. Considering sport level variation, student-athletes who competed at higher level of performance showed higher scores than those of lower competitive levels for SM and CM dimensions. Lastly, there was no substantial variation in the motivation scores by student-athlete status.

Supplementary Table 3 (available at https://osf.io/cpwdv/) presents the SAMSAQ-PT estimates and uncertainty ( $90 \%$ confidence intervals).

[Insert figures 1, 2, and 3]

\section{Discussion}

We tested the SAMSAQ-PT construct validity by exploring the variation in the motivation scores considering student-athletes' individual, sport, and academic characteristics. There was substantial variation in the SAMSAQ-PT dimension scores by sex, university type, and sport level. The observed substantial gender-related variation in SM and CM could be due to lower dual career support for Brazilian female athletes and limited economic opportunities for top-level women's sport (Harrison et al., 2020). Coherently, during the academic path, the highest effect scores for AM emerged for female student-athletes, who might emphasize more their academic career concerning their sports career (Tekavc et al., 2015). These findings urge implementing sex-related dual careers at the sport and public education level, as envisaged by the Europeanfunded Collaborative Partnership "Dual Career for Women Athletes" (DONA). Consistent with previous studies (Guidotti et al., 2013; Lupo et al., 2017), the sport level resulted as a predictor of CM and SM scores. It seems reasonable to assume that student-athletes competing at a higher level might be more motivated to pursue their sports career compared to their pairs competing at lower levels, whereas it is plausible to assume that AM is linked to the academic motivation of the athlete (Gaston-Gayles, 2005; Guidotti et al., 2013; Lupo et al., 2015; Lupo et al., 2017).

Peer) reviewing PDF | (2021:03:58830:2:0:NEW 30 Jun 2021) 
355

356

357

358

359

360

361

362

363

364

365

366

367

368

369

370

371

372

373

374

375

376

377

378

379

380

381

382

383

384

385

386

387

388

389

390

391

392

393

394

To our knowledge, this is the first study investigating the student-athletes' motivation related to university type (e.g., public and private). Indeed, the social context of universities shapes the opinions of student-athletes (Druckman et al., 2014). The present findings highlight that Brazilian student-athletes' sports and education commitments vary between public and private universities. Concerning their peers from public universities, in the present models, student-athletes from private universities have a high probability of scoring higher values for CM and SM, probably due to dual-career financial and logistic support (Aquilina, 2013; Aquilina \& Henry, 2010). Enrolling 75\% of the total undergraduate students (Instituto Nacional de Estudos e Pesquisas Educacionais Anísio Teixeira, 2018), private Brazilian universities might employ marketing strategies incorporating student-athletes to increase the media attention leveraging the universities' profile (Harrison et al., 2010; Teixeira, 2010). Unlike private universities, Brazilian public universities do not require tuition fees and offer academic undergraduate and graduate degrees (e.g., master's and Ph.D.), opportunities for research, and internships, which might contribute to future professional advantages (Cecić Erpič et al., 2004). Thus, compared to their counterparts enrolled in private universities, student-athletes attending public universities could show lower SM when prioritizing their academic careers to prepare for future job opportunities (Amara et al., 2004; McKenna \& Dunstan-Lewis, 2004). Additionally, the Brazilian university sports facilitating sub-elite athletes' participation in public institutions are still in a developmental phase (Starepravo et al., 2010).

At the international level, there is a call for awareness of the role and responsibilities of dual-career actors (Condello et al., 2019), and the implementation of support policies for studentathletes is a priority of the European Union (Aquilina \& Henry, 2010; European Commission, 2012; European Parliament, 2015, 2017). Indeed, student-athletes should be informed about their rights, and the implementation of dual-career counseling has been strongly recommended to help them manage their academic and sports commitment (Hansen \& Sackett, 1993; Martin, 2005; López de Subijana et al., 2015). During the academic path, a recognized student-athlete status could allow the monitoring of the academic and sport progresses to individualize necessary dualcareer support. Although this status has been considered crucial to influence substantially the athlete's motivation to achieve an academic degree, especially for those determined to pursue a sports career, the present models do not support this hypothesis. Thus, it is likely needed to take a step back and review the conceptions of support based on interactions at and across environmental levels (Knight et al., 2018).

\section{Study four}

To explore the SAMSAQ-PT scores' sensitiveness, we considered repeated measures across an academic year was considered for the analysis of changes in motivation scores adjusted for sex, sport level, student-athlete status, and type of university. Given attrition expected in longitudinal observations, a Bayesian multilevel regression modeling and poststratification were used to predict the changes applied to all observations in the cross-sectional data. Bayesian multilevel regression and poststratification allow for improved estimations of small and sparse group data 
395 (in the present study, the longitudinal observations) and consequently predicts a target population 396 (in the present study, the cross-sectional observations) (Gelman \& Little, 1997; Park, Gelman, \& 397 Bafumi, 2004; Kennedy \& Gelman, 2020).

398

399

400

401

402

403

404

405

406

407

408

409

410

411

412

413

414

415

416

417

418

419

420

421

422

423

424

425

426

427

428

429

430

\section{Methods}

\section{Participants}

This study included 99 female and 35 male student-athletes enrolled in public $(\mathrm{n}=68)$ and private $(\mathrm{n}=66)$ Brazilian universities competing at international $(\mathrm{n}=12)$, national $(\mathrm{n}=39)$, state $(\mathrm{n}=25)$, and university ( $\mathrm{n}=58)$ levels. Data were collected with a one-year interval (2018 and 2019) during the state and national University championships.

\section{Data analysis}

Due to the presence of non-representative and imbalanced data, with hierarchical sources of variation or cross-classified nesting, the first step of the analytical approach was to fit multilevel models to the repeated measures data, allowing for the possibility of varying intercepts (i.e., baseline values) and slope (changes in individuals outcomes across the period of observation) by sex, student-athlete status, sport level, and type of university (for individual $i$, with indexes, $s, a$, $l$, and $u$ for, sex, student-athlete status, sport level, and type of university, respectively).

Considering the homogeneity of slopes by group, we fitted varying intercepts models with measurement time as a population-level effect. The multilevel model specification was as follows:

$$
y_{i}=\beta^{0}+\text { time }_{i}+\alpha_{s[i]}^{\text {sex }}+\alpha^{\text {student }- \text { athlete status }}+\alpha_{a[i]}^{\text {sport level }}+\alpha_{l[i]}^{\text {type of }} \underset{u[i]}{\text { university }}+\epsilon_{i}
$$

$$
\begin{aligned}
& \alpha_{s[i]}^{s e x} \sim N\left(0, \sigma_{s e x}^{2}\right), \text { for } s=1,2 . \\
& \alpha^{\text {student - athlete status }} \sim N\left(0, \sigma_{\text {student }- \text { athlete status }}\right) \text {, for } a=1,2 . \\
& \alpha_{l[i]}^{\text {sport level }} \sim N\left(0, \sigma_{\text {sport level }}^{2}\right), \text { for } l=1,2,3,4 . \\
& \alpha^{\text {type of }} \underset{u[i]}{\text { university }} \sim N\left(0, \sigma_{\text {type of university }}\right), \text { for } u=1,2 \text {. } \\
& \epsilon_{i} \sim N\left(0, \sigma_{y_{i}}^{2}\right)
\end{aligned}
$$

Then, the multilevel model estimates to predict the student-athletes' outcomes for groups defined in a poststratification dataset (i.e., measurement time, sex, student-athlete status, sport level, and type of university) were used. The poststratification table considers the cross-sectional data sample in this research as a target population. The poststratification table has an observation corresponding to each group defined for all combinations of the variables included in the model. In this study, the poststratification table included two repeated measurement levels, two sex levels, two student-athlete status levels, four sport levels, and two type of university levels, encompassing 64 rows $(2 \times 2 \times 2 \times 4 \times 2)$, including the sample size, in each group. After 
431 predicting the outcome variable for each group, estimates for measurement time with the

432 subgroup sample sizes were aggregated. Hence, the method allowed full use of all available data

433 to interpret the changes in student-athletes' motivation scores, adjusted for individual and

434 contextual characteristics. The model's estimates were regularized by using normal prior $(0,10)$

435 for population-level effect (intercept) and normal priors $(0,1)$ for group-level effects. Four chains

436 for 4,000 iterations with 1,000 burn-in iterations were run. The models were obtained using the

437 "brms” package (Bürkner, 2017).

438

439

440

\section{Results}

441

Predicted changes for SM, AM, and CM, after an academic year and adjusted for sex, type of university, sport level, and student-athletes' status are summarized in Figure 4. The influence of the individual and context characteristics was similar at the baseline and after and academic year. The post-stratified predictions for the total sample showed a trend of stability for all studentathlete motivation scores after an academic year, although there was a slight decrease in SM.

447

\section{Discussion}

449 We explored the student-athletes' motivation scores' sensitiveness across an academic year adjusted for sex, sport level, student-athlete status, and type of university. The results showed

451

452

453

454

455

456

457

458

459

460

461

462

463

464

465

466

467

468

469

470
[Insert figure 4] that student-athletes' motivation was stable across an academic year. In addition to limited variations observed across an academic year, uncertainty estimates were narrow after adjusting for sex, type of university, sport level, and student-athletes' status. These findings suggest that SAMSAQ-PT may be sensitive to track individuals changes over time. Although sports burnout was beyond the aim of the present study, decreased SM over time could be considered an indicator in interpreting dual career paths (Sorkkila et al., 2018), monitoring university dualcareer social contexts, or highlighting the needs of the dual-career implementation. The present predictive models indicate that the student-athletes' motivation could be influenced by the university context and how it cares about the requirements necessary to combine sport and academic commitments, especially when the academic requirements increase towards graduation. Based on the present longitudinal observations, the university sports contexts appear not to motivate student-athletes in their dual-career. Future studies focusing on support dualcareer policies could contribute to a sound interpretation of how institutions and managers deal with dual-career needs and services.

While in some countries, a well-systematized relationship between education and highperformance sport seems to determine the high motivation for an athletic career (Fortes et al., 2010; Simons et al., 1999), in European countries with distinct academic and sports systems, athletes are confronted with the choice of one of the two paths (European Commission, 2016). In Brazil, sports and educational systems are also distinct. In line with the development of university sports in Brazil, the awareness of the importance of supporting student-athletes dual- 
471 career increases (Guidotti et al., 2015; Stambulova \& Wylleman, 2019). While the present results

472 showed that student-athletes' motivation does not vary substantially across an academic year,

473 further longitudinal studies are needed to verify whether student-athletes are motivated towards a

474 dual-career untill their graduation. Indeed, cooperation between the academic and sports sectors

475 needs to be implemented for providing a supportive entourage for student-athletes.

476

\section{General discussion}

478 Considering that contexts influence the motivation towards dual-career of athletes and its

479 specificities in the development of guidelines to support student-athletes, and contribute to the

480 discussion on student-athletes' motivation (European Commission, 2012). The four experimental

481 studies encompassing BEFA, BCFA, and Bayesian multilevel regression models in cross-

482 sectional and longitudinal samples validated the psychometric structure and assessed the

483 sensitiveness of the SAMSAQ-PT instrument in a Brazilian context. Furthermore, the present

484 study presents methodological and practical implications. Indeed, cross-cultural variation and

485 confidence in the measured outcomes need to be established when adapting a psychometric scale 486 to a specific context.

487 Given the debate concerning the limitations and inappropriateness of null-hypothesis 488 testing, "statistical significance" and using p-values (Amrhein \& Greenland 2018, McShane, Gal 489 et al. 2019), the Bayesian statistics was deemed appropriate to provide a natural approach to 490 account for di $\square$ erent sources of inferential uncertainty (Kennedy \& Gelman 2020). Furthermore, 491 in the present study, the analytical approach to validate a questionnaire using a multilevel 492 modeling framework was considered appropriate to deal with common limitations of sports 493 psychology research, such as noisy measurements, between-individuals heterogeneity, complex 494 interactions between outcomes, and non-representative and imbalanced samples. The present 495 data showed that the questionnaire scales were sensitive to the individual and contextual 496 characteristics of the target population. Overall, the SAMSAQ-PT was a valid questionnaire for 497 the Brazilian context and may be extended to Portuguese-speaking countries. Lastly, the study 498 contributes to the propositions of career construction theories within university-student athletes 499 (Rudolph et al., 2019). Finally, data, codes and supplementary tables from the present research 500 are available at (https://osf.io/cpwdv/).

$501 \quad$ Regarding the practical implications, the present findings provide a view of the Brazilian 502 educational system and how it might be related to the student-athletes' motivation towards a dual 503 career. In particular, relevant higher education stakeholders should cooperate in implementing 504 regulations and policies fostering the development of dual-career for supporting student-athletes 505 in combining sports and academic commitments. Considering that no variation was observed 506 across an academic year, sports counseling or dual-career developmental programs should focus 507 on first-year students. Likely highly motivated student-athletes since their first year of university 508 will be engaged in a dual-career during higher education and beyond. Local and national sports 509 policies should also consider the need to decrease sex-related differences by providing more 
510 opportunities for women in sports and increase media attention for public universities and 511 women in sports.

512 Despite the advantages of a Bayesian multilevel modeling approach and a large sample of 513 student-athletes, the present study presents some limitations. Only student-athletes competing in 514 the University Sports Games were considered even though university students could compete in 515 other Brazilian championships. Thus, further studies are needed to provide insights into the sport 516 and academic development. Another limitation pertains to the lack of information on individual 517 and contextual characteristics such as chronological age, academic course and year, or type of 518 sport (team or individual) that prevented further in-depth analyses. Future research is envisaged

519 to investigate the student-athlete background and transition from high school to higher education

520

521

522 and their motivation to choose public or private universities related to their dual-career.

523

524

525

526

527

528

529

530

531

532

533

534

535

536

537

538

539

540

541

542

543

544

545

546

547

548

549

550

551

\section{Conclusions}

The present study assessed the validity of the Portuguese version of the SAMSAQ-IT/A for the Brazilian context and potentially can add to advance the understanding of the student-athletes' motivation for a dual-career in other Portuguese-speaking countries. The use of the Bayesian estimations for psychometric analysis, multilevel regression and poststratification added to the analysis of the validity of psychometric scales in the study of student-athletes, which might suggest the revision of scales used in other countries. Based on the findings, it is possible to assume that individual and contextual characteristics need to be considered when investigating dual-career motivation. Based on the Brazilian context, the academic (public and private universities) and the sport (sport level) contexts may substantially impact student-athletes' motivation. Although the student-athletes' motivation overtime did not present substantial variation, the motivation decreased with time. Thus, the present results highlight the need to monitor the athlete's motivation towards sports and academic achievements untill graduation to develop optimal dual-career paths. The final translated and validated version of the SAMSAQ$\mathrm{PT}$ is presented in the Appendix 1.

\section{References}

Amara, M., Aquilina, D., Henry, I., \& PMP Consultants (2004). Education of young sportspersons. European Commission Directorate-General Education and Culture.

Amrhein, V. \& Greenland, S. (2018). Remove, rather than redefine, statistical significance. Nature Human Behaviour, 2(1), 4. https://doi.org/10.1038/s41562-017-0224-0

Aquilina, D. (2009). "Degrees of Success: Negotiating Dual Career Paths in Elite Sport and University Education in Finland, France and the UK." Loughborough University.

Aquilina, D. (2013). A Study of the Relationship Between Elite Athletes' Educational Development and Sporting Performance. The International Journal of the History of Sport, 30(4), 374-392. https://doi.org/10.1080/09523367.2013.765723.

Aquilina, D., \& Henry, I. (2010). Elite athletes and university education in Europe: a review of policy and practice in higher education in the European Union Member States. International Journal of Sport Policy, 2(1), 25-47. https://doi.org/10.1080/19406941003634024.

Peer] reviewing PDF | (2021:03:58830:2:0:NEW 30 Jun 2021) 
552 Brasil (1941). Decreto-lei n ${ }^{0} 3.199$, de 14 de abril de 1941. Estabelece as bases de organização

553

554

555

556

557

558

559

560

561

562

563

564

565

566

567

568

569

570

571

572

573

574

575

576

577

578

579

580

581

582

583

584

585

586

587

588

589

590

591

592

593

594

595 dos desportos em todo o país. Diário. https://legis.senado.leg.br/norma/528286.

Brasil (1998). Lei n ${ }^{\circ} 9.615$, de 24 de março de 1998. Institui normas gerais sobre desporto e dá outras providências. https:/www2.camara.leg.br/legin/fed/lei/1998/lei-9615-24-marco1998-351240-publicacaooriginal-1-pl.html.

Breslin, G., Shannon, S., Haughey, T., Sarju, N., Neill, D., Leavey, G., \& Lawlor, M. (2019). Athlete and Nonathlete Intentions to Self-Manage Mental Health: Applying the Integrated Behavior Change Model to the State of Mind Program. Journal of Applied Sport Psychology, 1-15. https://doi.org/10.1080/10413200.2019.1629547.

Bürkner, P. (2017). brms: An R Package for Bayesian Multilevel Models using Stan. Journal of Statistical Software, 80(1). https://doi.org/10.18637/jss.v080.i01.

Can, S., Van de Schoot, R., \& Hox, J. (2014). Collinear Latent Variables in Multilevel Confirmatory Factor Analysis: A Comparison of Maximum Likelihood and Bayesian Estimations. Educational and Psychological Measurement, 75(3), 406-427. https://doi.org/10.1177/0013164414547959

Carpenter, B., Gelman, A., Hoffman, M. D., Daniel Lee, Goodrich, B., Betancourt, M., Brubaker, M., Guo, J., Li, P., \& Riddell, A. (2017). Stan: A Probabilistic Programming Language. Journal of Statistical Software, 76(1), 32. https://doi.org/10.18637/jss.v076.i01.

Cartigny, E., Fletcher, D., Coupland, C., \& Taylor, G. (2019). Mind the Gap: A Grounded Theory of Dual Career Pathways in Sport. Journal of Applied Sport Psychology, 1-22. https://doi.org/10.1080/10413200.2019.1654559.

Carvalho, R. A. T., \& Hass, C. M. (2015). Conflito na legislação brasileira referente à escolarização de seus jovens atletas Revista de Estudios e Investigación en Psicologia y Educación, extr(12). https://doi.org/10.17979/reipe.2015.0.12.421.

Cecić Erpič, S., Wylleman, P., \& Zupančič, M. (2004). The effect of athletic and non-athletic factors on the sports career termination process. Psychology of sport and exercise, 5(1), 45-59. https://doi.org/10.1016/S1469-0292(02)00046-8.

Condello, G., Capranica, L., Doupona, M., Varga, K., \& Burk, V. (2019). Dual-career through the elite university student-athletes' lenses: The international FISU-EAS survey. PLoS One, 14(10), e0223278. https://doi.org/10.1371/journal.pone.0223278.

Conti, G., Frühwirth-Schnatter, S., Heckman, J. J., Piatek, R. (2014). Bayesian Exploratory Factor Analysis. Journal of Econometrics, 183(1), 31-57. https://doi.org/10.1016/j.jeconom.2014.06.008.

Druckman, J. N., Gilli, M., Klar, S., \& Robison, J. (2014). The role of social context in shaping student-athlete opinions. PloS One, 9(12), 1-14. https://doi.org/10.1371/journal.pone.0115159.

Dunn, J. G. H., Gotwals, J. K., \& Dunn, J. C. (2005). An examination of the domain specificity of perfectionism among intercollegiate student-athletes. Personality and Individual Differences, 38(6), 1439-1448. https://doi.org/10.1016/j.paid.2004.09.009.

European Commission. (2012). Guidelines on Dual Careers of Athletes: Recommended Policy Actions in Support of Dual Careers in High-Performance Sport. European Union. https://ec.europa.eu/assets/eac/sport/library/documents/dual-career-guidelinesfinal_en.pdf. 
596

597

598

599

600

601

602

603

604

605

606

607

608

609

610

611

612

613

614

615

616

617

618

619

620

621

622

623

624

625

626

627

628

629

630

631

632

633

634

635

636

637

638

639

640

641

European Commission. (2016). Study on the minimum quality requirements for dual career services. European Union. https://op.europa.eu/it/publication-detail//publication/e06e5845-0527-11e6-b713-01aa75ed71a1.

European Parliament. (2015). EU sport policy. An overview. Brussels: European Parliamentary Research Service. http://www.europarl.europa.eu/RegData/etudes/IDAN/2015/565908/EPRS_IDA(2015)56 5908_EN.pdf.

European Parliament. (2017). European Parliament Resolution of 2 February 2017 on an Integrated Approach to Sport Policy: Good Governance, Accessibility and Integrity. $\mathrm{http}: / /$ www.europarl.europa.eu/sides/getDoc.do?type=TA\&reference=P8-TA-20170012\&language $=\mathrm{EN} \&$ ring $=\mathrm{A} 8-2016-0381$.

Gomez, J., Bradley, J., \& Conway, P. (2018): The challenges of a high performance student athlete, Irish Educational Studies, 37(3), 329-349. https://doi.org/10.1080/03323315.2018.1484299.

Ferdinand, N., \& Czernochowski, D. (2018). Motivational Influences on Performance Monitoring and Cognitive Control Across the Adult Lifespan. Frontiers in Psychology, 26(9), 1018. https://doi.org/10.3389/fpsyg.2018.01018.

Fernandes, A., Moreira, L., \& Gonçalves, C. (2019). Student-athletes identity and motivation towards dual career: the context matters. Kinesiologia Slovenica, 25(2), 15-29.

Fortes, P. C., Rodrigues, G., \& Tchantchane, A. (2010). Investigation of academic and athletic motivation on academic performance among university students. International Journal of Trade, Economics and Finance, 1(4), 367-372. https://doi.org/7763/IJTEF.2010.V1.65.

Gao, Y., Kennedy, L., Simpson, D., \& Gelman, A. (2020). Improving multilevel regression and poststratification with structured priors. Bayesian Analysis, 26. https://doi.org/10.1214/20-BA1223

Gaston-Gayles, J. (2005). The factor structure and reliability of the student athletes' motivation toward sports and academics questionnaire (SAMSAQ). Journal of College Student Development, 46(3), 317-327. https://doi.org/10.1353/csd.2005.0025.

Gaston-Gayles, J., \& Baker, A. (2015). Opportunities and Challenges for First-Year StudentAthletes Transitioning from High School to College. New Dir Stud Leadersh, 2015(147), 43-51. https://doi.org/10.1002/yd.20142.

Gelman, A., Carlin, J. B., Stern, H. S., Dunson, D. B., Vehtari, A., \& Rubin, D. B. (2013). Bayesian Data Analysis. Chapman \& Hall/CRC Press.

Gelman, A., \& Hill, J. (2007). Data analysis using regression and multilevel/hierarchical models Cambridge University Press.

Gelman, A., \& Little, T. C. (1997). Poststratification into many categories using hierarchical logistic regression. Survey Methodology, 23(2), 127-135.

Gelman, A., \& Shalizi, C. R. (2013). "Philosophy and the practice of Bayesian statistics." British Journal of Mathematical and Statistical Psychology, 66(1), 8-38. https://doi.org/10.1111/j.2044-8317.2011.02037.x.

Guedes, D. P., \& Netto, J. E. (2013). Sport participation motives of young Brazilian athletes. Perceptual and Motor Skills, 117(3), 742-759. https://doi.org/10.2466/06.30.PMS.117x33z2.

Guidotti, F., \& Capranica, L. (2013). Le motivazioni verso sport, istruzione e carriera sportiva degli studenti-atleti italiani. In A. M. Pioletti \& N. Porro (Eds.), Lo sport degli europei: cittadinanza, attività, motivazioni. Franco Angeli.

Peer] reviewing PDF | (2021:03:58830:2:0:NEW 30 Jun 2021) 
642 Guidotti, F., Cortis, C., \& Capranica, L. (2015). Dual Career of European student-athletes: a

643 systematic litaruture review. Kinesiologia Slovenica, 21(3), 5-20.

644

645

646

647

648

649

650

651

652

653

654

655

656

657

658

659

660

661

662

663

664

665

666

667

668

669

670

671

672

673

674

675

676

677

678

679

680

681

682

683

684

685

Guidotti, F., Minganti, C., Cortis, C., Piacentini, M. F., Tessitore, A., \& Capranica, L. (2013). Validation of the Italian version of the Student Athletes' Motivation toward Sport and Academics Questionnaire. Sport Sciences for Health, 9(2), 51-58. https://doi.org/10.1007/s11332-013-0145-X.

Hansen, J.-I. C., \& Sackett, S. A. (1993). Agreement between College Major and Vocational Interests for Female Athlete and Non-athlete College Students. Journal of Vocational Behavior, 43(3), 298-309. https://doi.org/10.1006/jvbe.1993.1049.

Harrison, G. E., Vickers, E., Fletcher, D., \& Taylor, G. (2020). Elite female soccer players' dual career plans and the demands they encounter. Journal of Applied Sport Psychology, 1-22. https://doi.org/10.1080/10413200.2020.1716871.

Harrison, K., Rasmussen, J., Connolly, C., Janson, N., Bukstein, S., \& Parks, C. (2010). Diggin' Deeper into the Culture of Revenue Sports. Journal for the Study of Sports and Athletes in Education, 4(3), 325-332. https://doi.org/10.1179/ssa.2010.4.3.325.

Herdman, M., Fox-Rushby, J., \& Badia, X. (1997). 'Equivalence' and the translation and adaptation of health-related quality of life questionnaires. Quality of Life Research, 6(3), 237-247. https://doi.org/10.1023/a:1026410721664.

Hofstede, G., Garibaldi de Hilal, A. V., Malvezzi, S., Tanure, B., \& Vinken, H. (2010). Comparing regional cultures within a country: Lessons from Brazil. Journal of CrossCultural Psychology, 41(3), 336-352. https://doi.org/10.1177/0022022109359696.

Houlihan, B. (2005). Public sector sport policy developing a framework for analysis. International review for the sociology of sport, 40(2), 163-185.

Instituto Nacional de Estudos e Pesquisas Educacionais Anísio Teixeira. (2018). Sinópse estatística da educação superior 2017. http://inep.gov.br/sinopses-estatisticas-daeducacao-superior.

Joshanloo, M., Lepshokova, Z. K., Panyusheva, T., Natalia, A., Poon, W.-C., Yeung, V. W.-1., Sundaram, S., Achoui, M., Asano, R., Igarashi, T., Tsukamoto, S., Rizwan, M., Khilji, I. A., Ferreira, M. C., Pang, J. S., Ho, L. S., Han, G., Bae, J., \& Jiang, D.-Y. (2014). CrossCultural Validation of Fear of Happiness Scale Across 14 National Groups. Journal of Cross-Cultural Psychology, 45(2), 246-264. https://doi.org/10.1177/0022022113505357.

Kennedy, L., \& Gelman, A. (2020). Know your population and know your model: Using modelbased regression and poststratification to generalize findings beyond the observed sample. arXiv:1906.11323v2 [stat.AP]

Keshtidar, M., \& Behzadnia, B. (2017). Prediction of intention to continue sport in athlete students: A self-determination theory approach. PLoS One, 12(2). https://doi.org/10.1371/journal.pone.0171673.

Knight, C., Hardwood, C. G. \& Sellars, P. A. (2018). Supporting adolescent athletes' dual careers: The role of an athlete's social support network. Psychology of Sport \& Exercise, 38, 137-147. https://doi.org/10.1016/j.psychsport.2018.06.007.

Kruschke, J. K., \& Liddell, T. M. (2018). Bayesian data analysis for newcomers. Psychonomic Bulletin \& Review, 25(1), 155-177. https://doi.org/10.3758/s13423-017-1272-1

Lee, M.D.; Wagenmakers, E. J. Bayesian Cognitive Modeling: A Practical Course; Cambridge University Press: Cambridge, UK, 2013.

Peer) reviewing PDF | (2021:03:58830:2:0:NEW 30 Jun 2021) 
686

687

688

689

690

691

692

693

694

695

696

697

698

699

700

701

702

703

704

705

706

707

708

709

710

711

712

713

714

715

716

717

718

719

720

721

722

723

724

725

726

727

728

729
Liang, X. (2020). Prior Sensitivity in Bayesian Structural Equation Modeling for Sparse Factor Loading Structures. Educational and Psychological Measurement, 80(6), 1025-1058. https://doi.org/10.1177/0013164420906449

López de Subijana, C., Barriopedro, M., \& Conde, E. (2015). Supporting dual career in Spain: Elite athletes' barriers to study. Psychology of Sport and Exercise, 21, 57-64. https://doi.org/10.1016/j.psychsport.2015.04.012.

Lupo, C., Guidotti, F., Goncalves, C., Moreira, L., Doupona Topic, M., Bellardini, H., Tonkonogi, M., Colin, A., \& Capranica, L. (2015). Motivation towards dual career of European student-athletes. European Journal of Sport Science, 15(2), 151-160. https://doi.org/10.1080/17461391.2014.940557.

Lupo, C., Mosso, C. O., Guidotti, F., Cugliari, G., Pizzigalli, L., \& Rainoldi, A. (2017). Motivation toward dual career of Italian student-athletes enrolled in different university paths. Sport Sciences for Health, 13(3), 485-494. https://doi.org/10.1007/s11332-0160327-4.

Lupo, C., Tessitore, A., Capranica, L., Rauter, S., \& Doupona-Topic, M. (2012). Motivation for a dual-career: Italian and Slovenian student-athletes. Kinesiologia Slovenica, 18(3), 4756.

Martin, S. B. (2005). High School and College Athletes' Attitudes Toward Sport Psychology Consulting. Journal of Applied Sport Psychology, 17(2), 127-139. https://doi.org/10.1080/10413200590932434.

McKenna, J., \& Dunstan-Lewis, N. (2004). An action research approach to supporting elite student-athletes in higher education. European Physical Education Review, 10(2), 179198. https://doi.org/10.1177/1356336X04044070.

McShane, B. B., Gal, D., Gelman, A., Robert, C., \& Tackett, J. L. (2019). Abandon Statistical Significance. The American Statistician, 73(sup1), 235-245. https://doi.org/10.1080/00031305.2018.1527253.

Merkle, E; C., \& Rosseel, Y. (2018). blavaan: Bayesian Structural Equation Models via Parameter Expansion. Journal of Statistical Software, 85(4), 1-30. https://doi.org/10.18637/jss.v085.i04.

Monteiro, D., Cid, L., Marinho, D. A., Moutão, J., Vitorino, A., Bento, T. (2017). Determinants and reasons for dropout in swimming-systematic review. Sports (Basel), 5(3), 50. https://doi.org/10.3390/sports5030050.

Montenegro-Montenegro, E. (2020). Improving the guidelines to conduct multigroup invariance test in Bayesian SEM. (Dissertation), Texas Tech University, Texas.

Open Science Collaboration (2015). Estimating the reproducibility of psychological science. Science, 349(6251), aac4716. https://doi.org/10.1126/science.aac4716

Park, D. K., Gelman, A., \& Bafumi, J. (2004). Bayesian Multilevel Estimation with Poststratification: State-Level Estimates from National Polls. Political Analysis, 12(4), 375-385.

Park, S., Hong, S., \& Lee, M. (2015). Validation of the student athletes' motivation towards sports and academics questionnaire to Korean student-athletes. Journal of exercise rehabilitation, 11(4), 220. https://doi.org/10.12965/jer.150202.

Peeters, C. F. W. (2012). Bayesian exploratory and confirmatory factor analysis: Perspectives on constrained-model selection. Utrecht: Utrecht University. 
730

731

732

733

734

735

736

737

738

739

740

741

742

743

744

745

746

747

748

749

750

751

752

753

754

755

756

757

758

759

760

761

762

763

764

765

766

767

768

769

770

771

772

773

774
Quinaud, R. T., Fernandes, A., Goncalves, C. E., \& Carvalho, H. M. (2020). Student-Athletes' Motivation and Identity: Variation Among Brazilian and Portuguese University StudentAthletes. Psychological Reports, 123(5), 1703-1723. doi: 10.1177/0033294119892885

Quinaud, R. T., Gonçalves, C. E., Capranica, L., \& Carvalho, H. M. (2020). Factors Influencing Student Athletes' Identity: A Multilevel Regression and Post-stratification Approach. Perceptual and Motor Skills.

R Core Team. (2018). R: A Language and Environment for Statistical Computing. R Foundation for Statistical Computing. https://www.r-project.org.

Ryan, R., Bradshaw, E., \& Deci, E. (2019). A History of Human Motivation Theories. In R. Sternberg \& W. Pickren (Eds.), The Cambridge Handbook of the Intellectual History of Psychology. Cambridge University Press.

Ryba, T. V., Ronkainen, N. J., \& Selänne, H. (2015). Elite athletic career as a context for life design. Journal of Vocational Behavior, 88, 47-55. https://doi.org/10.1016/j.jvb.2015.02.002.

Schweizer, G. \& Furley, P. (2016). Reproducible research in sport and exercise psychology: The role of sample sizes. Psychology of Sport and Exercise, 23, 114-122. https://doi.org/10.1016/j.psychsport.2015.11.005.

Singer, J. D. \& Willett, J. B. (2003). Applied longitudinal data analysis: modeling change and event occurrence. Oxford; New York, Oxford University Press.

Simons, H., Van Rheenen, D., \& Covington, M. (1999). Academic motivation and the student athlete. Journal of College Student Development, 40, 151-162.

Sorkkila, M., Ryba, T., Selanne, H., \& Aunola, Kainsa. (2018). Development of school and sport burnout in adolescent student-athletes: a longitudinal mixed-methods study. Journal of Research on Adolescence, 30 (s1), 115-133. https://doi.org/10.1111/jora.12453.

Stambulova, N. B., \& Alfermann, D. (2009). Putting culture into context: Cultural and cross cultural perspectives in career development and transition research and practice. International journal of sport and exercise psychology, 7(3), 292-308. https://doi.org/10.1080/1612197X.2009.9671911.

Stambulova N. B., Ryba T. V., \& Henriksen, K. (2020). Career development and transitions of athletes: the International Society of Sport Psychology Position Stand Revisited, International Journal of Sport and Exercise Psychology, https://doi.org/10.1080/1612197X.2020.1737836.

Stambulova, N., \& Wylleman, P. (2015). Dual career development and transitions. Psychology of Sport and Exercise, 21, 1-3. https:// doi.org/10.1016/j.psychsport.2015.05.003.

Stambulova, N., \& Wylleman, P. (2019). Psychology of athletes' dual careers: A state-of-the-art critical review of the European discourse. Psychology of Sport and Exercise, 42, 74-88. https://doi.org/10.1016/j.psychsport.2018.11.013.

Starepravo, F. A., Reis, L. J. A., Mezzadri, F. M., \& Marchi Jr, W. (2010). Esporte universitário brasileiro: uma leitura a partir de suas relações com o Estado. Revista Brasileira de Ciências do Esporte, 31(3), 131-148.

Su, C. T., \& Parham, L. D. (2002). Generating a valid questionnaire translation for cross-cultural use. American Journal of Occupational Therapy, 56(5), 581-585. https://doi.org/10.5014/ajot.56.5.581.

Sullivan, P., Murphy, J., \& Blacker, M. (2020). The Psychometric Properties of the Multicomponent Mental Health Literacy Measure with a Sample of Student Athletes and 
775

776

777

778

779

780

781

782

783

784

785

786

787

788

789

790

791

792

793

794

795

796

797

798

799

800

801

802

803

804

805

806

807

808

809

810

811

812

813

814 among Brazilian student-athletes' by sex, type of university, sport level, and student-athlete 815 status.

Student Athletic Therapists. Journal of Applied Sport Psychology, 1-14. https://doi.org/10.1080/10413200.2019.1702119.

Teixeira, M. (2010). Influência dos eventos esportivos no posicionamento competitivo de uma universidade particular: estudo de caso UNISANTA. (Mestrado), Universidade Católica de Santos.

Tekavc, J., Wylleman, P., Cecić-Erpič, S. (2015). Perceptions of dual career development among elite level swimmers and basketball players. Psychology of Sport and Exercise, 21, 27-41. https://doi.org/10.1016/j.psychsport.2015.03.002.

Van de Schoot, R., Winter, S. D., Ryan, O., Zondervan-Zwijnenburg, M., \& Depaoli, S. (2017). A systematic review of Bayesian articles in psychology: The last 25 years. Psychological Methods, 22(2), 217-239. https://doi.org/10.1037/met0000100.

Van Hoorn, A. (2015). Individualist-Collectivist Culture and Trust Radius:A Multilevel Approach. Journal of Cross-Cultural Psychology, 46(2), 269-276. https://doi.org/10.1177/0022022114551053.

Wasserstein, R. L., Schirm, A. L., \& Lazar, N. A. (2019). Moving to a World Beyond "p < 0.05". The American Statistician, 73(sup1), 1-19. https://doi.org/10.1080/00031305.2019.1583913.

Wu, A. M. S., Lai, M. H. C., \& Chan, I. T. (2014). Coaching Behaviors, Satisfaction of Needs, and Intrinsic Motivation Among Chinese University Athletes. Journal of Applied Sport Psychology, 26(3), 334-348. https://doi.org/10.1080/10413200.2014.888107.

[Insert Appendix 1 about here]

\section{Tables' and figures captions:}

Table 1. Bayesian exploratory factor analysis (posterior means) of the Portuguese adapted version of the Student-Athletes' Motivation Toward Sports and Academics Questionnaire. Figure 1. Posterior values for Sport Motivation dimension by sex, type of universities, sport levels and student-athletes' status (67\% and 90\% credible intervals).

Figure 2. Posterior values for Academic Motivation dimension by sex, type of universities, sport levels and student-athletes' status (67\% and 90\% credible intervals).

Figure 3. Posterior values for Career Motivation dimension by sex, type of universities, sport levels and student-athletes' status (67\% and 90\% credible intervals).

Figure 4. Predicted changes for Sport Motivation, Academic Motivation and Career Motivation, after an academic year, adjusted for sex, type of university, sport level and student-athletes' status ( $90 \%$ credible intervals).

Supplementary table 1. Items means and standard deviation.

Supplementary table 2. Bayesian confirmatory factor loadings.

13 Supplementary table 3 . Estimates ( $90 \%$ confidence intervals) of SAMSAQ-PT factors responses 


\section{Table 1 (on next page)}

Bayesian exploratory factor analysis (posterior means) of the Portuguese adapted version of the Student-Athletes' Motivation Toward Sports and Academics Questionnaire. 
1 Table 1. Bayesian exploratory factor analysis (posterior means) of the Portuguese adapted version

2 of the Student-Athletes' Motivation Toward Sports and Academics Questionnaire.

\begin{tabular}{lcc}
\hline \multicolumn{1}{c}{ Item } & Factors \\
\hline \multicolumn{1}{c}{ 1. I am confident that I can achieve a high-grade point average this year } \\
(3.0 or above) & AM & CM \\
\hline $\begin{array}{l}\text { 2. Achieving a high level of performance in my sport is an important goal } \\
\text { for me this year }\end{array}$ & 5.02 \\
\hline 3. It is important to me to learn what is taught in my courses & \\
\hline 4. I am willing to put in the time to earn excellent grades in my courses & 5.61 \\
\hline $\begin{array}{l}\text { 5. Within an academic environment, I find it more challenging to face } \\
\text { difficult tasks }\end{array}$ & 5.19 \\
\hline 6. For me, studies are important to achieve knowledge and skills & 4.42 \\
\hline 7. I will be able to use what is taught in my courses in different aspects of \\
my life outside of school
\end{tabular}

8. I chose to play my sport, because it is something I am interested in as a career

9. For me, it is important to train seriously to improve my performance

4.77

10. I chose (or will choose) my major, because it is something I am interested in as a career

4.93

11. Earning a high-grade point average (27/30 or above) is not an important goal for me this year

$2.70^{*}$

12. It is important to me to learn the skills and strategies taught by my coaches

5.24

13. It is important for me to do better than other athletes in my sport

14. The time I spend engaged in my sport is enjoyable to me

15. It is worth the effort to be an exceptional athlete in my sport

16. The achievement of a degree is important to enrich my knowledge

17. In sport, I find stimulating those situations requiring high

performances and being difficult to perform

4.23

18. During the years, I compete in my sport, completing a college degree is not a goal for me

19. I am confident that I can be a star performer on my team this year

4.23

4.61

20. My goal is to make it to the professional level or the Olympics in my sport

21. Situations that allow me to test my capacities stimulate me

4.94

22. I am confident that I can make it to an elite level in my sport

(Professional/Olympics)

23. I am confident that I can earn a college degree

$2.09 *$

4.12

24. I will be able to use the skills I learn in my sport in other areas of my life outside of sports

5.63

25. Achieving high performances in my sport is not an important goal for me this year

26. For me, it is important to achieve high performances and not to make mistakes

27. I am willing to put in the time to be outstanding in my sport

4.62

28. The content of most of my courses is interesting to me

4.80

29. It is important for me to obtain a degree, because it will help me to find a job 
30. It is not worth the effort to earn excellent grades in my courses $1.78^{*}$

$3 \mathrm{SM}=$ Sport Motivation, $\mathrm{AM}=$ Academic Motivation, $\mathrm{CM}=$ Career Motivation .

$4 *$ Items excluded. 
Figure 1

Posterior values for Sport Motivation dimension by sex, type of universities, competitive levels and student-athletes' status (67\% and $90 \%$ credible intervals). 

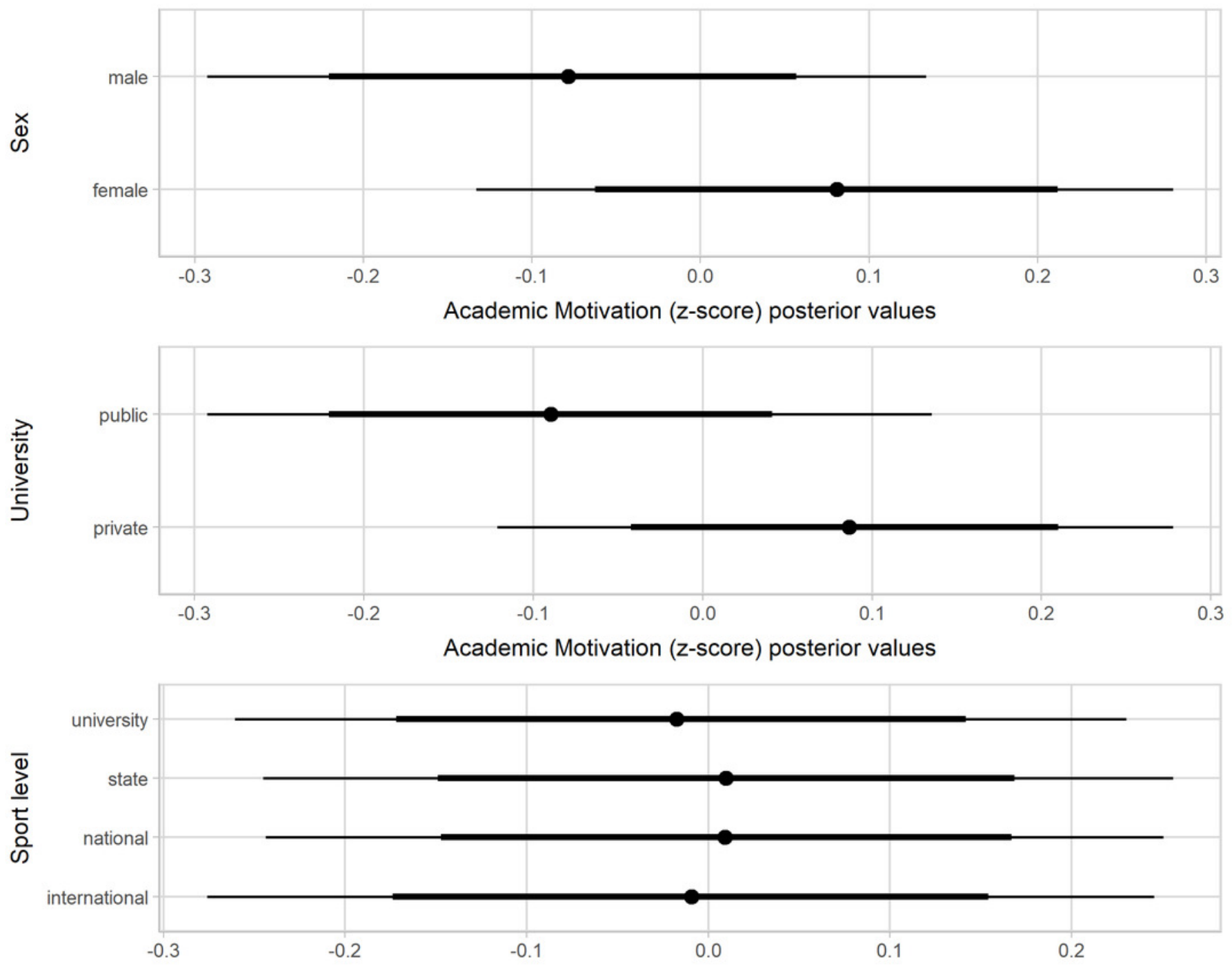

Academic Motivation (z-score) posterior values

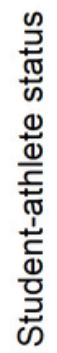

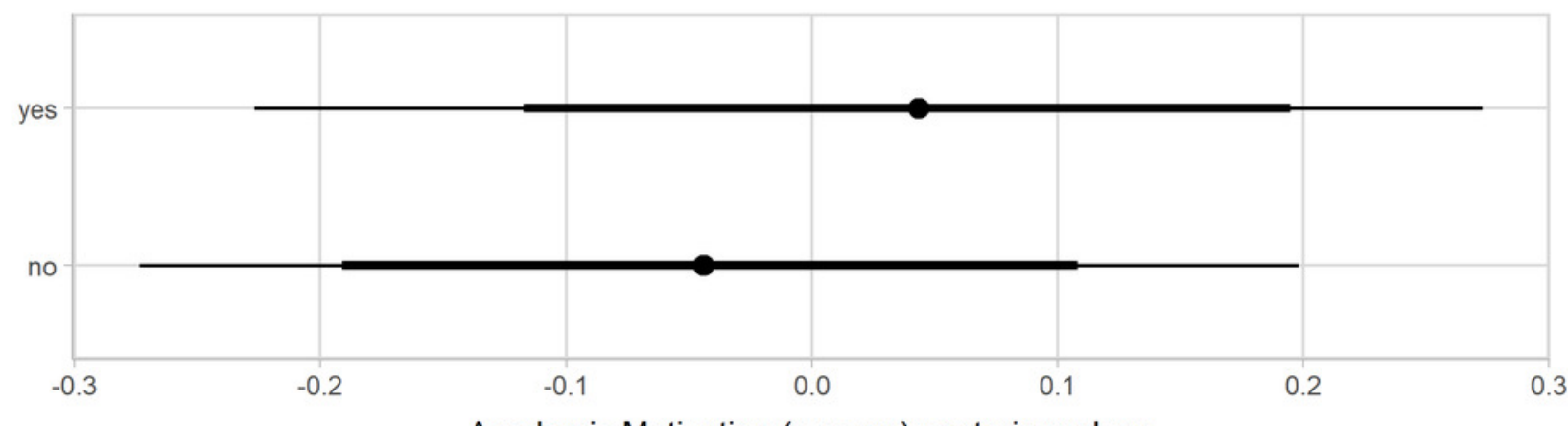

Academic Motivation (z-score) posterior values 
Figure 2

Posterior values for Academic Motivation dimension by sex, type of universities, competitive levels and student-athletes' status ( $67 \%$ and $90 \%$ credible intervals). 

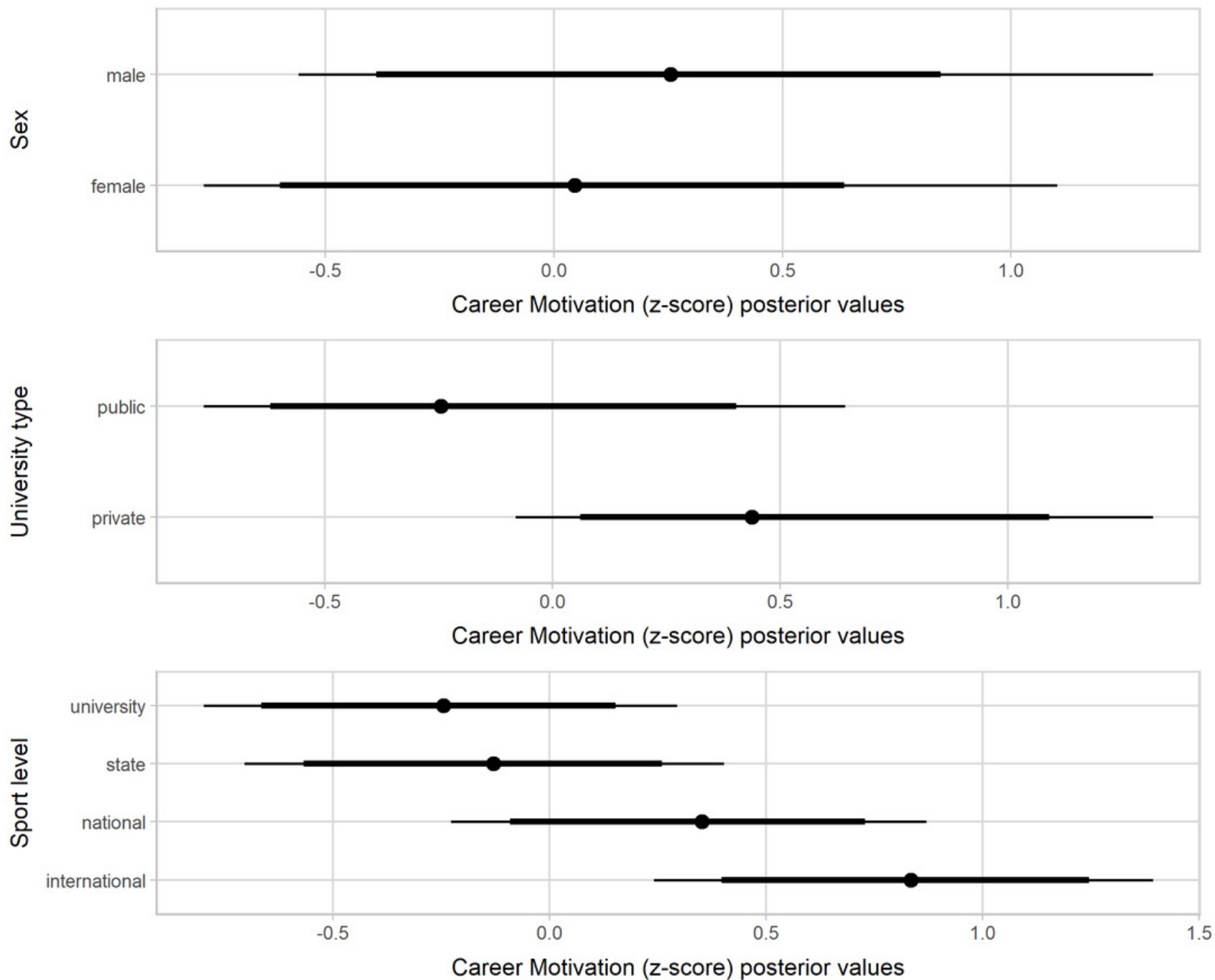

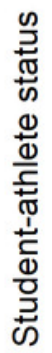

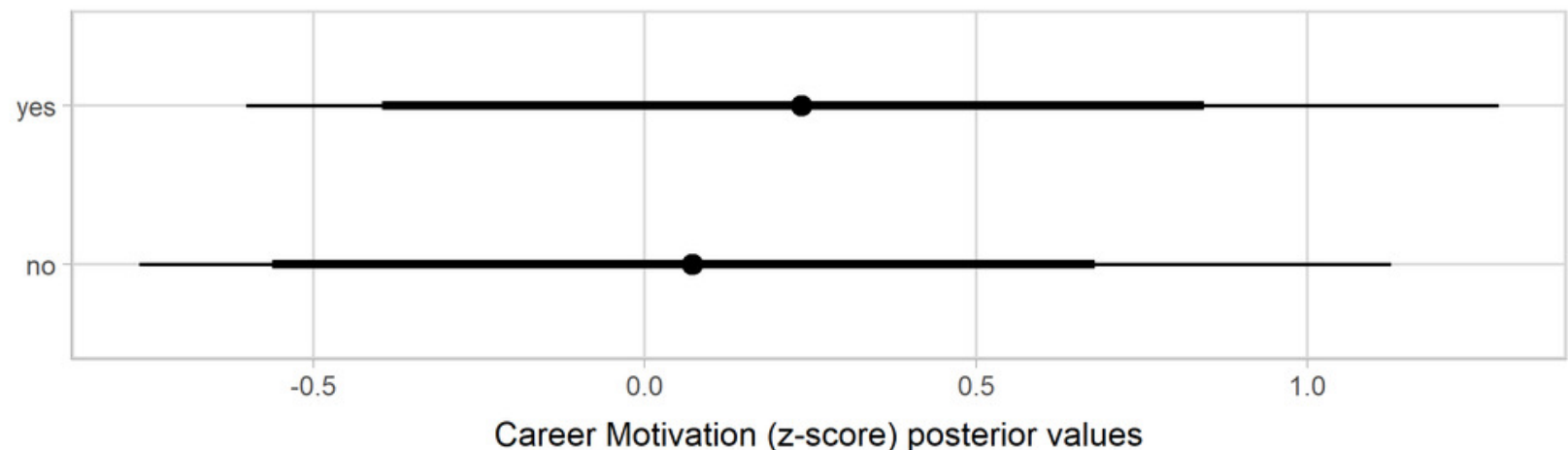

Career Motivation (z-score) posterior values 
Figure 3

Posterior values for Career Motivation dimension by sex, type of universities, competitive levels and student-athletes' status ( $67 \%$ and $90 \%$ credible intervals). 

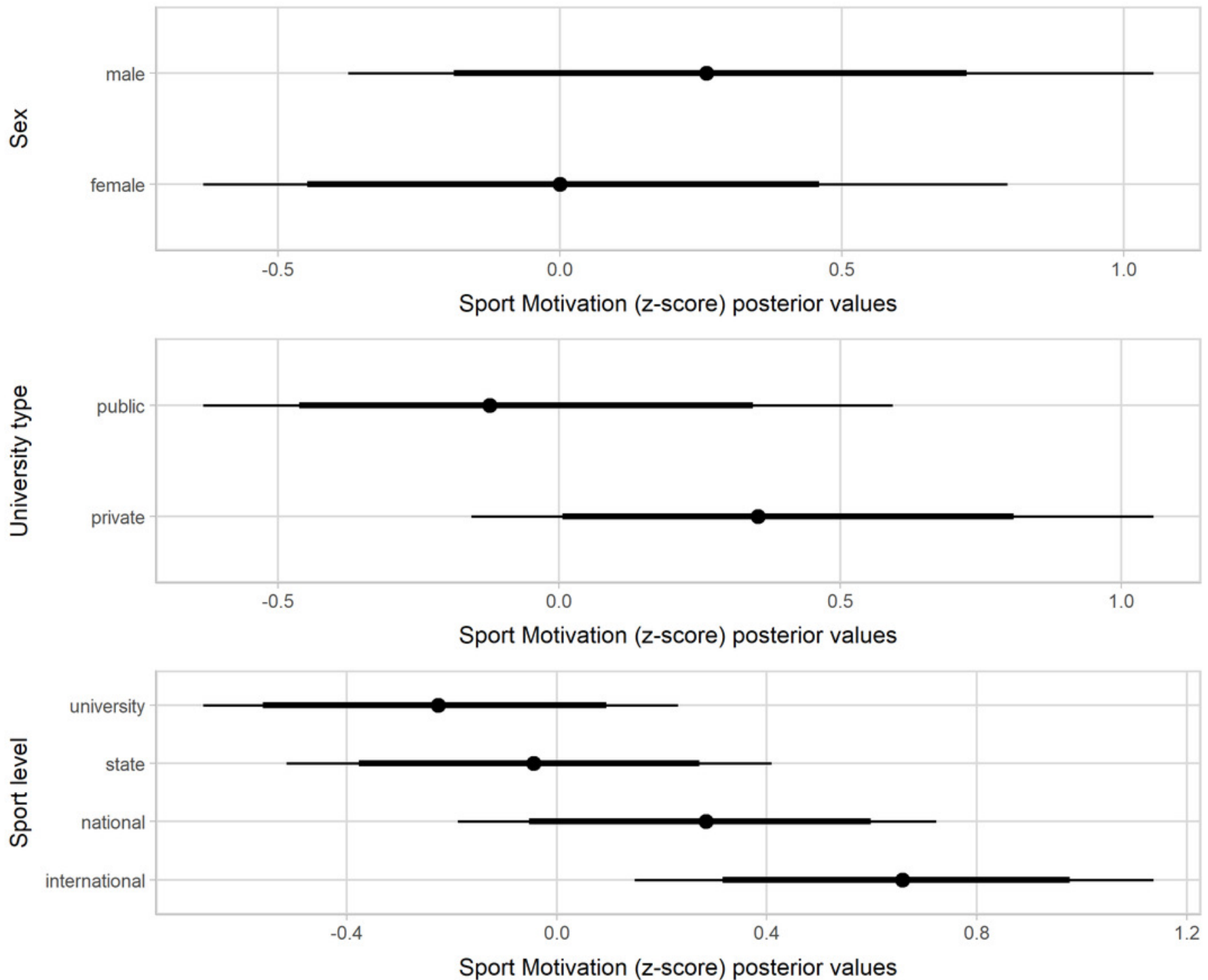

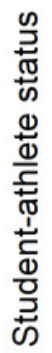

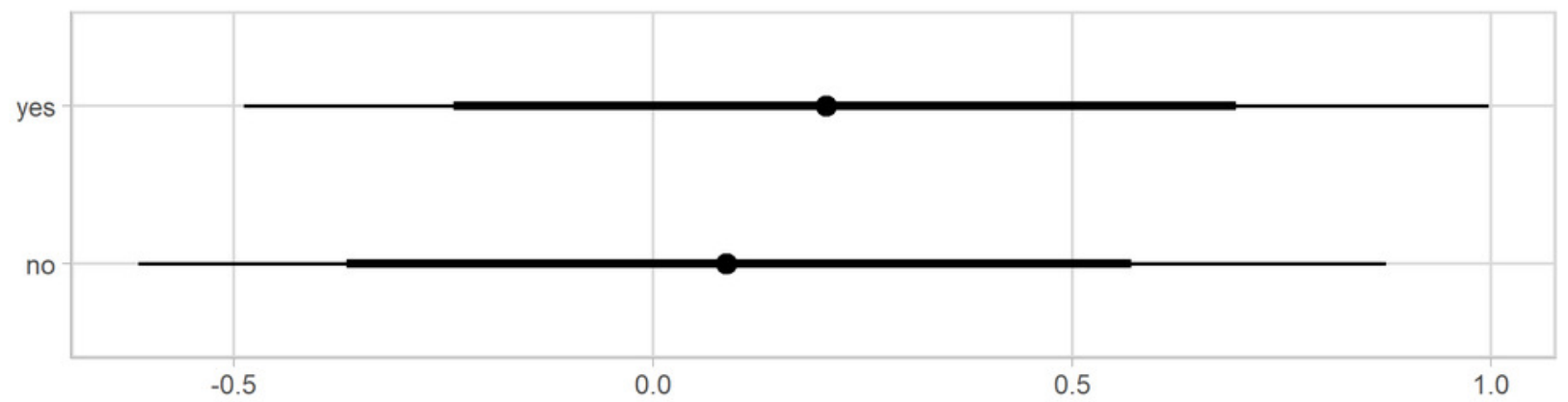

Sport Motivation (z-score) posterior values 


\section{Figure 4}

Predicted changes for Sport Motivation, Academic Motivation and Career Motivation, after an academic year, adjusted for gender, university type, competitive level and student-athletes' status ( $90 \%$ credible intervals).

Posterior values for Career Motivation dimension by sex, type of universities, competitive levels and student-athletes' status (67\% and $90 \%$ credible intervals). 


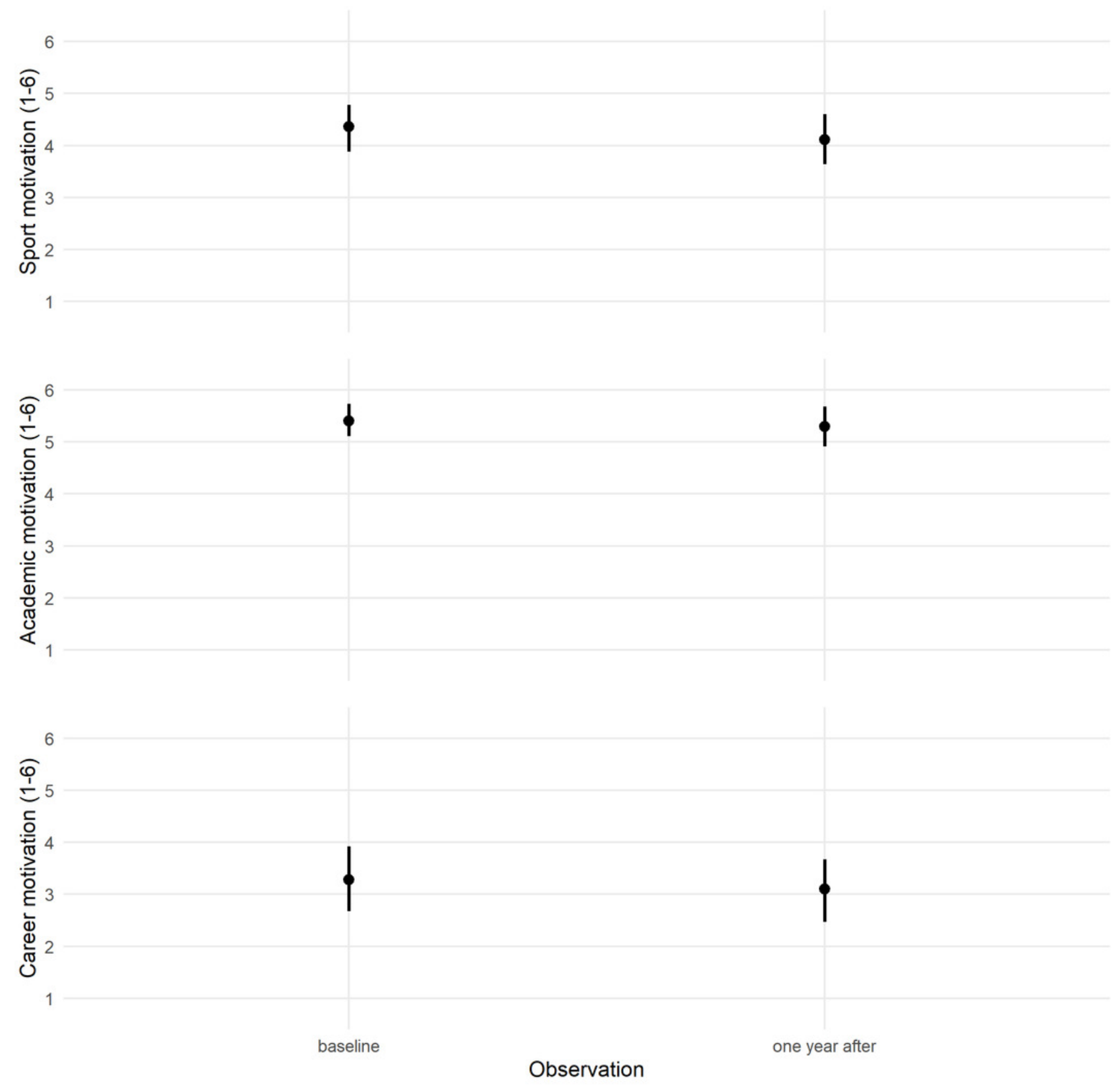

